\title{
Prise de position sur la Cigarette électronique
}

Société Suisse de Pneumologie (SSP), Société Suisse de Pneumologie pédiatrique (SSPP)
Correspondance:

Dr Macé M. Schuurmans Klinik für Pneumologie Universitätsspital Zurich Rämistrasse 100 CH-8091 Zürich

mace.schuurmans[at]usz.ch

PD Dr Jürg Barben Ostschweizer Kinderspital CH-9006 St. Gallen

juerg.barben[at]kispisg.ch
$\mathrm{Vu}$ que la cigarette électronique (e-cigarette) peut servir de système d'administration de nicotine à ses utilisateurs et utilisatrices, tout en reproduisant les caractéristiques typiques d'une cigarette de tabac, la Société Suisse de Pneumologie (SSP) et la Société Suisse de Pneumologie pédiatrique (SSPP) appellent les autorités compétentes et l'OFSP, à faire valoir leur mandat légal et à soumettre ce produit à une réglementation efficace. Une telle réglementation doit comprendre les exigences suivantes et prendre en considération certains aspects complémentaires:

- Les SSP/SSPP recommandent de maintenir en vigueur l'actuelle interdiction des e-cigarettes et des liquides contenant de la nicotine, jusqu'à ce qu'une clarification de l'utilité par rapport aux risques soit établi. Les données actuellement à disposition sont insuffisantes.

- La confédération doit considérer les cigarettes électroniques avec ou sans nicotine en tant que produits de tabac. Les SSP/SSPP demandent une être exclu pour la cigarette électronique ou qui pour le plupart des produits actuellement en vente doit être attendu (mesure de prévention contre le tabagisme).

- Les interdictions et restrictions de publicité et de sponsoring en vigueur pour les produits du tabac doivent s'appliquer de la même manière aux cigarettes électroniques. Les SSP/SSPP demandent une loi fédérale d'interdiction de publicité et du sponsoring pour tous les produits du tabac et des cigarettes électroniques (interdiction de la publicité).

- Les emballages des cigarettes électroniques doivent être munis de mises en gardes, similaires en taille et en contenu à celles qui sont obligatoires pour les produits de tabac combustibles. Les utilisateurs/utilisatrices devraient être informés sur les risques encourus par un langage clair, sans équivoque. Là, où des données sur les risques font défaut ou sont controversées, les

\section{Nous recommandons de maintenir en vigueur l'actuelle interdiction des e-cigarettes.}

interdiction de la distribution sur tout le territoire suisse de cigarettes électroniques et de liquides avec ou sans nicotine à des personnes âgées de moins de 18 ans. Cette exigence devrait valoir pour tous les produits du tabac sur le territoire national (protection des enfants et des mineurs). De même, la vente par Internet et l'importation des cigarettes électroniques et des liquides sont à réglementer de manière stricte.

- Les e-cigarettes et les liquides contenant de la nicotine devraient être frappés d'un impôt équivalent à celui des cigarettes traditionnelles et autres produits du tabac (impôt sur le tabac).

- L'utilisation dans l'espace public des cigarettes électroniques devrait être soumis aux mêmes restrictions que celle des produits de tabac combustibles: L'utilisation de la cigarette électronique (avec ou sans nicotine) dans l'espace public fermé devrait être interdite, en analogie avec la réglementation pour la protection contre la fumée passive, sur la base de l'Art. 14 de la loi sur les aliments, selon laquelle des biens de consommation dans leur utilisation normale ou usuelle ne doivent pas nuire à la santé, ce qui ne peut pas consommateurs doivent être informés sur le manque de données fiables concernant l'innocuité du produit.

- Des affirmations implicites et explicites par les fabricants de cigarettes électroniques concernant la santé ou la sécurité du produit devraient être soumises aux exigences d'évidence scientifique au même titre que des prétentions similaires pour d'autres produits.

- Le contenu des cartouches destinées aux cigarettes électroniques doit être déclaré, régulé et contrôlé. Le contenu en nicotine d'une cigarette électronique ne doit pas dépasser le contenu en nicotine correspondant à 40 cigarettes de tabac combustibles. La quantité de nicotine administrée devrait être uniforme pour toutes les cartouches. Les cartouches doivent être munies d'une sécurité pour enfants. L'OFSP doit exiger des fabricants de e-cigarettes les mêmes principes de bonne fabrication, valables pour d'autres produits régulés, comme par ex. numéro de charge, emballages de sécurité, etc.

- Vu que la nicotine est une drogue qui rend dépendant ses utilisateurs/utilisatrices (Neurotoxine) 


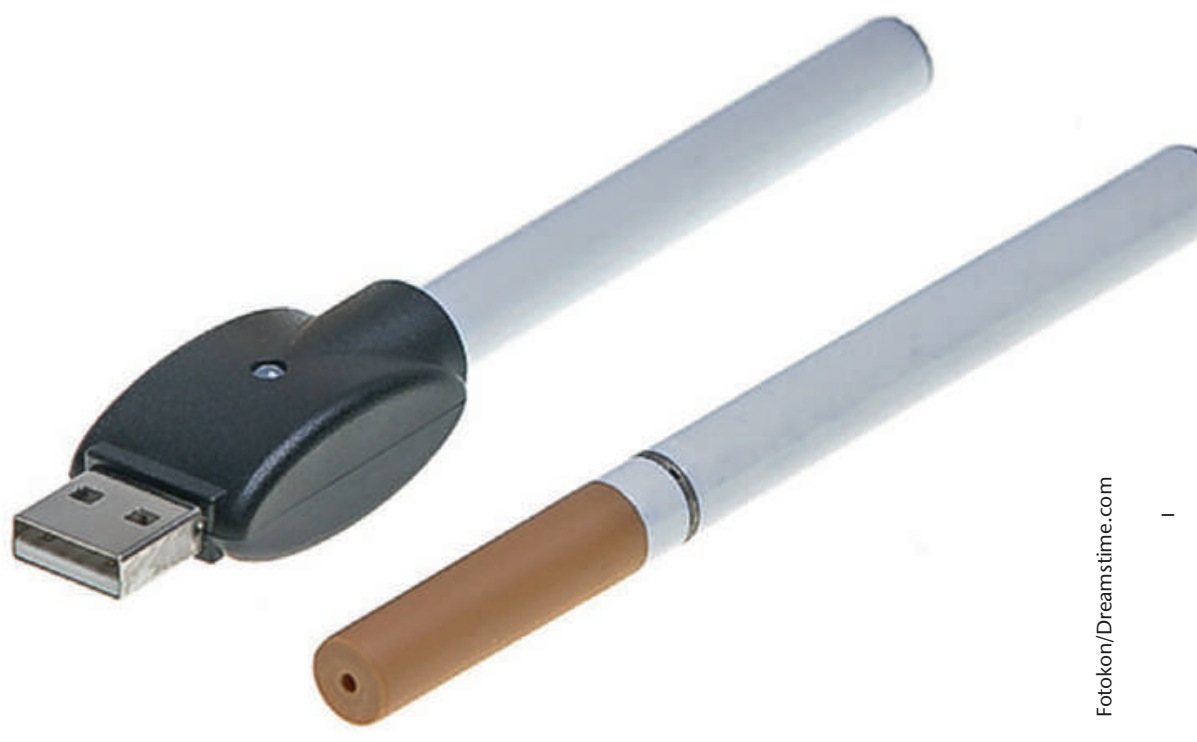

Le contenu des cartouches destinées aux cigarettes électroniques doit être déclaré, régulé et contrôlé.

et que le degré de l'addiction est dépendant de la pharmacocinétique du système d'administration, les qualités du système d'administration devraient être étudiées, rendues publiques et contrôlées régulièrement, afin de garantir la constance du produit sur la durée.

- Les e-cigarettes sont parfois promus en tant qu'aide à la désaccoutumance des fumeurs, malgré le fait que leur efficacité pour l'arrêt du tabagisme, est sujette à controverse parmi les experts.

\section{L'OMS, actuellement, déconseille la cigarette électro- nique en tant que moyen de désaccoutumance du tabagisme.}

conséquent le fait de s'être libéré de la nicotine. Cette définition doit être appliquée lorsqu'on évalue les mesures de désaccoutumance du tabagisme.

- Les SSP/ SSPP demandent, que les chercheurs et cliniciens, ainsi que les sociétés scientifiques et les publications scientifiques soutenus financièrement par des fabricants de cigarettes électroniques, rendent publiques ces relations et les possibles conflits d'intérêts, comme cela est exigé pour les relations avec l'industrie du tabac.

- Les SSP/ SSPP sont préoccupées par le fait, que le marché de la cigarette électronique est dominé à une large échelle par l'industrie du tabac. La similitude du produit avec la cigarette conventionnelle et sa promotion par la même industrie constitue un conflit fondamental.

\section{Références}

- Commission fédérale de prévention du tabagisme. Prise de position sur les cigarettes électroniques. Août 2012.

- American Thoracic Society: ATS Policy eCigarettes, http://news.thoracic.org/november-2013/the-advocate.php

- International Union Against Tuberculosis and Lung Disease. Position statement on electronic cigarettes or electronic nicotine delivery systems. Int J Tuberc Lung Dis. 2014;18(1):5-7.

- John Britton. Electronic cigarettes. Thorax published online August 12, 2013 doi: 10.1136/thoraxjnl-2013-203973.

- Bullen C et al. Electronic cigarettes for smoking cessation: a randomised controlled trial. Lancet. Published Online September 7, 2013 http://dx.doi. org/10.1016/ S0140-6736(13)61842-

- Fairchaild AL et al. The Renormalization of Smoking? E-Cigarettes and the Tobacco «Endgame». N Engl J Med. December 18; 2013.

- De Andrade M et al. Promotion of electronic cigarettes: tobacco marketing reinvented? BMJ. 2013;347:f7473 doi: 10.1136/bmj.f7473

L'OMS, actuellement, déconseille la cigarette électronique en tant que moyen de désaccoutumance du tabagisme. Le but d'un traitement de la dépendance du tabac, est l'abstinence durable et prouvée de la consommation de nicotine. L'accomplissement de l'arrêt du tabagisme est par
- Drummond MB et al. Electronic cigarettes. Potential harms and benefits. Ann Am Thorax Soc. 2014;11:23642.

- Elektrische Zigaretten - ein Überblick. Deutsches Krebsforschungsinstitut. Rote Reihe, Tabakprävention und Tabakkontrolle, Band 19. Deutsches Krebsforschungszentrum. Heidelberg; 2013. 